\title{
Estado neoliberal y gasto público en psicofármacos en el Chile contemporáneo
}

Neoliberal State and public expenditure of psychiatric drugs in contemporary Chile

\section{Estado neoliberal e gastos públicos com drogas psicotrópicas no Chile contemporâneo}

Juan Carlos Cea Madrid ORCID ID: 0000-0001-7790-8138 Centro de Estudios Locos, Chile

Autor referente: jcarlos.ceamadrid@gmail.com

Historia Editorial

Recibido: 18/03/2018

Aceptado: 24/10/2018

\section{RESUMEN}

El presente trabajo aborda el lugar del Estado en la concepción neoliberal de la salud mental en base al predominio del mercado farmacéutico. Para ello, se cuestiona la extensión de la atención ambulatoria y el aumento de la prescripción de psicofármacos como ejes articuladores de la política pública de salud mental en Chile. Junto con ello, se examina el rol del Estado neoliberal en base a la transferencia de recursos públicos al sector privado,

considerando los montos de licitaciones y laboratorios involucrados en el gasto público en psicofármacos en el período 2011-2017. Se concluye que el uso masivo de fármacos psiquiátricos es la respuesta preferencial del Estado para la atención de salud mental y que la obtención de beneficios económicos con recursos públicos por parte de la industria farmacéutica, debe ser rebatida desde una clave de lectura crítica y alternativa al neoliberalismo.

Palabras clave: Psicofármacos; Neoliberalismo; Estado subsidiario; Chile

\section{ABSTRACT}

The following article addresses the States place in the neoliberal conception of mental health on the basis of the pharmaceutical market's predominance. For that purpose, the extension of outpatient care and the increase of psychiatric drug prescriptions as key elements of mental 
health public policies in Chile. In addition, the role of the neoliberal state on the basis of transferring public resources to the private sector is examined, considering the amounts of bids and laboratories involved in public expenditure of psychiatric drugs during the period of 2011-2017. It is concluded that the massive use of psychiatric drugs is the State's preferred response for mental health care and the obtaining of economic benefits with public resources on the part of the pharmaceutical industry, must be contested using a critical and alternative analysis to neoliberalism.

Keywords: Psychiatric drugs; Neoliberalism; Subsidiary State; Chile

\section{RESUMO}

O presente trabalho aborda o lugar do Estado na concepção neoliberal de saúde mental a partir do predomínio do mercado farmacêutico. Para tanto, questiona-se a extensão do atendimento ambulatorial e o aumento da prescrição de drogas psicotrópicas como eixos articuladores da política pública de saúde mental no Chile. Junto com isso, o papel do Estado neoliberal é examinado com base na transferência de recursos públicos para o setor privado, considerando os montantes das propostas e laboratórios envolvidos nos gastos públicos com drogas psicotrópicas no período 2011 2017. Concluise que 0 uso generalizado de medicamentos psiquiátricos é a resposta preferida do Estado para os cuidados de saúde mental e obter benefícios econômicos de fundos públicos pela indústria farmacêutica deve ser desafiados a partir de uma leitura crítica chave e alternativa neoliberalismo

Palavras-chave: Drogas psicotrópicas; Neoliberalismo; Estado subsidiário; Chile

\section{Introducción}

n América Latina, diversas corrientes críticas y perspectivas alternativas han
posibilitado el avance del enfoque comunitario en salud mental. A pesar de ello, la influencia de estas iniciativas no ha logrado un retroceso significativo del modelo asilar. Por el contrario, la psiquiatría de orientación biológica ha articulado las prácticas de encierro tradicionales con nuevas formas de control de la subjetividad. En este contexto, los psicofármacos representan la extensión del manicomio en las sociedades contemporáneas (Cipriano, 2017).

Desde una mirada crítica, diversos investigadores de Europa y Norteamérica, han llamado la atención de la ciudadanía y los organismos públicos al afirmar que los fármacos psiquiátricos no son tan seguros, ni tan eficaces ni tan específicos como se 
presentan en el mercado, señalando que en ocasiones pueden ser una fuente más de problemas que de soluciones y generar más daño que beneficio, en particular, en su uso combinado y a largo plazo (Bentall, 2011; Gøtzsche, 2015; Moncrieff, 2013; Whitaker, 2015). En el plano sociocultural, la medicalización de la subjetividad tiene directa relación con la expansión de una mirada que comprende los malestares subjetivos como hechos naturales, que no dependen de las circunstancias sociales, sino problemas de tipo cerebral etiquetados bajo categorías psiquiátricas (Pérez-Soto, 2012). Para Moncrieff (2006), esta individualización y naturalización del malestar social, se articula con las políticas neoliberales, posibilitando la proliferación de etiquetas psiquiátricas y el explosivo aumento de personas diagnosticadas con "trastornos mentales", así como la expansión de los servicios de salud mental y el consumo de psicofármacos.

En Chile, las políticas públicas de salud mental se han desarrollado desde el Estado de acuerdo a un enfoque planteado como comunitario. El eje de la reforma ha consistido en la inyección de mayores recursos para la ampliación de las consultas de salud mental en centros de atención primaria y la organización de servicios ambulatorios con arraigo territorial, complementando las prácticas tradicionales de internación psiquiátrica de carácter público y privado. Junto con ello, la ampliación de la cobertura de atención se ha desarrollado de acuerdo a criterios neoliberales de eficiencia y racionalización del gasto, incentivando la externalización de servicios sanitarios y la privatización de la gestión hospitalaria.

De acuerdo a estas lógicas institucionales, el enfoque comunitario se ha entendido principalmente como un "modelo de gestión", sobre la base de la creación de una red de dispositivos orientados a desarrollar acciones sanitarias y asistenciales en el ámbito local, lo que se ha asociado al incremento del consumo de fármacos psiquiátricos en la población. Entre las escasas investigaciones que abordan este fenómeno, Jirón, Machado y Ruiz (2008) señalan que el consumo total de dosis diarias de 
antidepresivos por cada 1.000 habitantes sufrió el incremento de un $470 \%$ en 12 años, de 2,5 en 1992 a 11,7 en 2004. Este estudio, también detectó que el antidepresivo más consumido es la fluoxetina, representando el $59 \%$ del total de dosis diarias en 1992 y el $48 \%$ en 2004. Ese mismo año, un estudio sobre el consumo de psicofármacos en Santiago de Chile determinó que un $6,4 \%$ de la población encuestada estaba consumiendo algún psicofármaco al momento de la entrevista, especialmente benzodiazepinas (3,8\%) (Rojas, Fritsch, Galleguillos, Gaete \& Araya, 2004).

Al respecto, Galleguillos, Risco, Garay, González y Vogel (2003) y Olivera (2009) sostienen que la ingesta de benzodiacepinas entre pacientes consultantes en Atención Primaria de Salud (APS) alcanza entre 30-40\%, siendo mayoritariamente mujeres, de edad avanzada en contexto urbano. Junto con ello, Santibáñez (2013) determinó que el alprazolam es el ansiolítico más importado, distribuido y consumido en farmacia comunitaria en Chile entre el período 2007-2012.

En el marco institucional, la implementación en Chile de la Ley 19.966, que establece el Régimen de Garantías en Salud y Sistema de Garantías Explícitas (GES), ha establecido que a contar del año 2005, las personas que presentan un primer episodio de esquizofrenia cuentan con garantías de acceso y oportunidad a tratamiento, tanto en el sistema público como privado de salud. Al respecto, una evaluación de la implementación de la Guía Clínica GES para primer episodio de esquizofrenia, determinó que todos los pacientes que participaron del estudio recibieron tratamiento farmacológico con algún tipo de antipsicótico y la gran mayoría de ellos inició su tratamiento con risperidona (85,5\%) (Alvarado, 2006).

Por otra parte, según datos de la Encuesta Nacional de Salud 2009-2010, un 7,8\% de la población reportó consumir psicoanalépticos (estimulantes y antidepresivos) así como un 5,6\% psicolépticos (ansiolíticos, hipnóticos y antipsicóticos). El psicofármaco más consumido por la población chilena para uso global y uso exclusivamente crónico, 
es el clonazepam con un 2,1\%. Del mismo modo, este instrumento determinó en un 6,6\% la prevalencia de tratamiento farmacológico por depresión en la población encuestada (Ministerio de Salud, 2010). Considerando los estudios señalados, en Chile un significativo número de personas se han convertido en usuarios de servicios de salud mental y consumidores de psicofármacos en los últimos años.

Un componente asociado a la naturalización del consumo de medicamentos psiquiátricos en la actualidad, refiere al marco explicativo que afirma que los problemas de salud mental están causados por un desequilibrio químico que puede ser corregido por fármacos específicos. Al respecto, Whitaker (2015) señala:

Hemos llegado a pensar, como sociedad, que la psiquiatría ha conseguido hacer en los últimos cincuenta años grandes progresos en el tratamiento de la enfermedad mental, que los científicos están descubriendo las causas biológicas de los trastornos mentales y que las empresas farmacéuticas han desarrollado una serie de medicamentos eficaces para tratarlos. (p. 13)

Sin embargo, este panorama promisorio contrasta con la continua falta de evidencia sobre el origen biológico de los denominados "trastornos mentales" y la inexistencia de pruebas científicas sobre la efectividad de cualquier tipo de intervención psiquiátrica (Pérez-Soto, 2012).

A pesar de ello, el número de personas etiquetadas con diagnósticos psiquiátricos es cada vez más alto y el número de personas en situación de discapacidad por motivos psiquiátricos ha aumentado exponencialmente (Whitaker, 2015). A su vez, el discurso de la "enfermedad mental" se ha extendido más allá de las instituciones psiquiátricas y la producción de psicofármacos se ha expandido considerablemente en las últimas décadas. De acuerdo a esta clave de lectura, los psicofármacos representan un objeto de estudio relevante para comprender los vínculos de la salud mental y el neoliberalismo. En particular, es relevante analizar de manera crítica cómo se articulan los intereses económicos en torno a la producción, compra, distribución y consumo de 
psicofármacos en tanto mercancías, considerando el rol del Estado en la configuración y ampliación del mercado farmacéutico. A partir de ello, el presente trabajo se inscribe en los campos de interrelación e influencia del modelo neoliberal en su articulación con el Estado, en base a la exposición de elementos descriptivos de gasto público en psicofármacos en el Chile contemporáneo.

\section{Salud mental y neoliberalismo}

El predominio del discurso psiquiátrico en la sociedad contemporánea ha propiciado un poderoso consenso: los fármacos psiquiátricos funcionan y ayudan a las personas a llevar una vida relativamente normal (Whitaker, 2015). Sin embargo, en la medida que las causas biológicas de los "trastornos mentales" no han sido establecidas y los medicamentos psiquiátricos no han mostrado ser beneficiosos a partir de los propios criterios de la ciencia médica (Pérez-Soto, 2012), representa un desafío analizar bajo qué condiciones se ha construido el discurso dominante que señala que los psicofármacos enriquecen la vida de las personas y les permiten vivir más plenamente.

Como ya se ha señalado, los psicofármacos expresan la característica principal de la salud mental en los tiempos del libre mercado. En un escenario neoliberal, los valores del individualismo, el consumismo, la competitividad y la productividad, refuerzan la idea de responsabilidad de los sujetos sobre su propio bienestar y su involucramiento activo para afrontar dificultades de acuerdo a los parámetros de lo normal y lo deseable. En ese marco, adquiere sentido que un individuo aislado y autosuficiente acuda a la consulta del psiquiatra y acepte tomar psicofármacos, en la medida que se ha construido socialmente una determinada forma de concebir el proceso de buscar ayuda, solicitar atención, expresar su problema y pensarse a sí mismo, de acuerdo a las presiones e imposiciones de la narrativa neoliberal. De esta manera, en el campo sociocultural, la producción de significados en torno al consumo de psicofármacos, se 
imbrican con las fuerzas políticas y económicas arraigadas en los procesos de comercialización de estos medicamentos, como son los intereses comerciales de los laboratorios, cuya finalidad es obtener beneficios económicos y vender sus mercancías en el mercado.

Si bien es posible analizar ampliamente los factores sociales y culturales que articulan la relación psicofármacos y subjetividad en el neoliberalismo, en el marco de este trabajo se describirán algunos elementos de la influencia de los intereses comerciales en las prácticas asistenciales que sustentan la ampliación del mercado farmacéutico y la prescripción de psicofármacos en el campo de la salud mental.

En Chile, se ha advertido sobre el bajo presupuesto público de la salud mental en comparación a los países de la OCDE y en relación al gasto total de salud (Errázuriz, Valdés, Vöhringer \& Calvo, 2015). Del mismo modo, informes recientes destacan el preocupante aumento de los denominados "trastornos mentales" en la población económicamente activa, siendo el principal motivo de licencias laborales (Miranda, Alvarado \& Kaufman, 2012). De acuerdo a estas cifras, las "enfermedades mentales" serían la principal causa de pérdida de años de vida saludables, lo que implica un alto costo en términos de productividad. En este marco, la Organización Mundial de la Salud (OMS) ha llegado a afirmar que uno de los obstáculos para el desarrollo de América Latina es el bajo presupuesto que se destina para abordar las problemáticas de salud mental en la región. En consecuencia el Banco Mundial (BM), ha enfatizado el tema de salud mental como un elemento importante en la agenda del desarrollo y prioridad para las autoridades económicas y de salud de sus países miembros.

Siguiendo estos postulados, los planes y programas de atención de salud mental para la detección temprana y tratamiento oportuno de "enfermedades mentales", han sido las claves que sustentan la ampliación de la cobertura de atención ambulatoria en los últimos años en Chile. Esto ha implicado un incremento de la oferta de servicios de salud mental en la perspectiva de reducir la brecha entre necesidades de atención y el 
tratamiento recibido. Sin embargo, el mayor acceso de la población a tratamiento de salud mental, se ha traducido principalmente en un aumento de la cobertura de psicofármacos. Respecto a este último punto, el Estado asume la siguiente relación: se promoverá la salud mental de la población si cuenta con mayor acceso a fármacos psiquiátricos. Este vínculo no es fortuito y da cuenta de un entramado ideológico en clave neoliberal que sostiene el discurso hegemónico de la psiquiatría.

Si bien bajo el neoliberalismo es responsabilidad exclusiva del individuo cuidar su salud, para el Estado aumentar la salud de la población adquiere importancia en la medida que se visualiza como una inversión: las personas serán poseedoras de mayor "capital humano", que podrán vender (intercambiar) en el mercado a mayor precio aumentando sus ingresos y mejorando la productividad (González-Guzmán, 2015). Del mismo modo, bajo el principio de "igualdad de oportunidades" las personas al obtener atención de salud podrán disminuir sus barreras de discapacidad e integrarse activamente en los circuitos productivos, por lo tanto, en adelante sus desigualdades se deberán solo al esfuerzo, el mérito individual y su capacidad de emprendimiento. Bajo estos principios, las políticas públicas de salud, y de salud mental en particular, están orientadas disminuir brechas de atención y no salen de la lógica del mercado, más bien, se comprenden como una inversión en capital o como una fuente de lucro (González-Guzmán, 2015).

Bajo estas orientaciones ideológicas, el Estado neoliberal promueve la mercantilización del derecho a la salud y la privatización de los servicios públicos, ampliando la competitividad del sistema y la expansión del mercado. A su vez, el sujeto neoliberal está llamado a conducirse como un "empresa de sí mismo", por lo tanto se ensalza la elección, la independencia y la responsabilidad de los individuos en lo que se refiere a su capacidad de acceder a prestaciones sanitarias y desarrollar sus capacidades en términos de adaptación a los parámetros de la sociedad capitalista. 
En este contexto, es relevante considerar que neoliberalismo no es "menos Estado". Por el contrario, el mercado es una construcción de la intervención estatal, cuya acción se orienta a preservar el marco institucional apropiado para el desarrollo de la propiedad privada individual y el libre mercado (Laval y Dardot, 2013). Un aspecto que profundiza el modelo neoliberal en el contexto chileno, es la funcionalización del Estado respecto del interés de los empresarios privados, introduciendo la lógica de las empresas privadas en la gestión de los servicios públicos y ampliando prácticas orientadas a privilegiar e incluso financiar directamente a empresas privadas en detrimento de sus propios servicios (Pérez-Soto, 2014). En particular, el debilitamiento sistemático de la infraestructura en salud pública, unido al sistema de licitaciones y concesiones a empresas privadas de servicios sanitarios, constituyen los mecanismos característicos de transferencia de fondos públicos al sector privado en el ámbito de la salud (Pérez-Soto, 2014). Bajo estos mecanismos, los gobiernos democráticos han ampliado la inversión y el gasto público en salud en base a procesos de externalización y privatización, enarbolando retóricas progresistas paralelas a la consistente profundización del modelo económico y social neoliberal (Pérez-Soto, 2015). De esta forma, la inversión pública en esta área prioritaria, ha implicado un aumento de los clientes de servicios de salud mental y una extensión del número de consumidores de psicofármacos.

Si bien se han enunciado diversas formas en que el Estado interviene para ampliar y fortalecer el mercado en el campo de la salud mental, describir y analizar los diversos alcances del entramado público-privado y sus relaciones en un contexto neoliberal, exceden las posibilidades del presente artículo. En ese sentido, a continuación se analizará un ámbito acotado y particular en que opera la relación del Estado con la industria farmacéutica en el ámbito de la salud mental en Chile, a través del gasto público en psicofármacos. 


\section{Metodología}

Para aproximarse a la inversión monetaria en psicofármacos en el sistema público de salud chileno, en febrero del 2018, a través del portal de acceso a la información pública se solicitó a la Central Nacional de Abastecimiento (CENABAST)—entidad encargada de realizar compras de insumos clínicos y medicamentos para hospitales, consultorios y centros de salud administrados por el Estado-información sobre las compras realizadas de fármacos antipsicóticos (clorpromazina, clozapina, flufenazina, haloperidol, olanzapina, quetiapina, risperidona, sulpirida y ziprasidona) y antidepresivos (amitriptilina, anfebutamona, citalopram, clomipramina, fluoxetina, imipramina, paroxetina, sertralina, trazodona y venlafaxina) en el período 2011-2017. Respecto al marco de análisis económico de los datos obtenidos, cabe señalar que el mercado farmacéutico en Chile se caracteriza por un ámbito público y uno privado. Ambos mercados comparten los mismos fármacos, laboratorios y marcas. Sin embargo, hay importantes diferencias de precio en cada uno de ellos. En el mercado público, la CENABAST, permite acceder a precios muy diferentes a los presentados al consumidor en farmacias privadas. Lo anterior se explica debido que la CENABAST desarrolla mecanismos de compra mediante licitaciones que involucran valores de oferta públicos y enormes volúmenes de productos, lo que le permite ejercer un poder de negociación en la transacción de medicamentos. Frente a ese sistema, los laboratorios optan por reducir sus posibles ganancias, bajar los precios y asegurar la venta, en la medida que la empresa que gana la licitación se lleva toda la demanda del Estado (Figueroa, 2015).

A pesar de estas ventajas y beneficios, la CENABAST sólo intermedia el $40 \%$ de las compras de medicamentos del sistema público de salud, en la medida que los hospitales y/o centros de salud también pueden utilizar licitaciones públicas o compras de trato directo con los proveedores. Al respecto, la CENABAST ofrece una lista pública de precios de los productos licitados, sin embargo, esta característica no es 
compartida por otros intermediarios, por lo tanto, existen dificultades para acceder a los precios pagados por otros compradores del sistema público (Centro Nacional de Farmacoeconomía [CENAFAR], 2013). De esta manera, los datos obtenidos de la CENABAST no representan la totalidad del gasto público en psicofármacos del Estado chileno, sin embargo, evidencian un aspecto claro y específico de la inversión estatal en este ámbito, expresando información relevante sobre la relación del sector público con el mercado farmacéutico en el campo de la salud mental.

De acuerdo a los datos obtenidos, a continuación se presentan los montos de licitaciones y laboratorios involucrados en las compras públicas de antipsicóticos y antidepresivos realizadas por CENABAST en el período 2011-2017.

\section{Gasto público en psicofármacos: más Estado es más mercado}

En base a la información recopilada sobre los montos de dinero invertido por el Estado a través de la CENABAST, para la compra de fármacos antipsicóticos y antidepresivos en el período 2011-2017, en la tabla 1, se observa un crecimiento del gasto público en ambas clases de psicofármacos en el período evaluado. El año 2011 las compras de antipsicóticos efectuadas por CENABAST correspondieron a \$496.013.600. El año 2017, el gasto público para este tipo de medicamentos ascendió a \$1.090.850.268. De esta forma, se observa un $119,9 \%$ de aumento de inversión monetaria en antipsicóticos del año 2011 al 2017. Por otra parte, el año 2011 las compras de antidepresivos por parte de CENABAST tuvieron un valor monetario de $\$ 649.857 .921$. El año 2017, el gasto público para esta clase de psicofármaco aumentó a $\$ 1.706 .086 .185$. De esta manera, se observa un $162,5 \%$ de incremento de dinero gastado en antidepresivos en el período 2011-2017. 
Tabla 1

Gasto público en antipsicóticos y antidepresivos (2011 - 2017)

Gasto público en psicofármacos (pesos chilenos)

\begin{tabular}{lll}
\hline & Antipsicóticos & Antidepresivos \\
2011 & 496.013 .600 & 649.857 .921 \\
2012 & 564.719 .150 & 744.719 .025 \\
2013 & 291.866 .500 & 736.279 .915 \\
2014 & 641.208 .260 & 775.007 .034 \\
2015 & 475.470 .649 & 997.220 .876 \\
2016 & 852.785 .735 & 1.813 .907 .540 \\
2017 & 1.090 .850 .268 & 1.706 .086 .185 \\
Total & 4.412 .914 .162 & 7.423 .078 .496 \\
\hline
\end{tabular}

Nota: Elaboración propia en base a datos obtenidos de la Central Nacional de Abastecimiento (CENABAST), Ministerio de Salud, Chile (Monto neto, no incluye impuesto al valor agregadoIVA, ni comisión de CENABAST).

En relación a las firmas de laboratorios que obtuvieron beneficios económicos a partir de transacciones con dinero público para la compra de antidepresivos y antipsicóticos entre los años 2011-2017, en la tabla 2 se observan las 15 empresas farmacéuticas que se adjudicaron licitaciones públicas para la venta de antidepresivos, en orden decreciente según el dinero obtenido por las transacciones realizadas. Los montos adjudicados entre los años 2011 y 2017 ascienden a \$7.423.078.496, alcanzando los primeros 4 laboratorios: LABORATORIO CHILE S.A., LABORATORIOS ANDROMACO S.A., OPKO CHILE S.A. y SOCOFAR S.A., beneficios económicos que 
ascienden a \$4.167.969.374, representando el 56,2\% del gasto público en antidepresivos del período evaluado.

Tabla 2

Gasto público en antidepresivos (2011 - 2017).

Laboratorios y montos de licitación (pesos chilenos)

Laboratorio Monto licitación

LABORATORIO CHILE S.A.

1.310.877.014

LABORATORIOS ANDROMACO S.A.

997.305.175

OPKO CHILE S.A.

955.457 .140

SOCOFAR S.A.

904.330 .045

LABORATORIOS SILESIA S.A.

860.933.305

MINTLAB Co. S.A.

724.850 .440

EUROFARMA CHILE S.A.

618.918 .509

RECBEN XENERICS FARMACEUTICA LTDA

543.172 .470

LABORATORIOS RECALCINE S.A.

144.577 .500

LABORATORIOS RIDER LTDA.

125.719 .260

INTERPHARMA S.A.

111.903 .930

FARMA STORAGE SPA

67.340 .000

NOVOFARMA SERVICE S.A.

42.871 .446

LABORATORIO MAVER S.A.

7.661 .500

TECNOFARMA S.A.

7.160 .762

Total

7.423.078.496

Nota: Elaboración propia en base a datos obtenidos de la Central Nacional de Abastecimiento (CENABAST), Ministerio de Salud, Chile (Monto neto, no incluye impuesto al valor agregadoIVA ni comisión de CENABAST). 
Por otra parte, respecto a las empresas farmacéuticas que obtuvieron utilidades financieras a partir de transacciones con dinero público para la compra de antipsicóticos, en la tabla 3 se presentan las 18 firmas de laboratorios que participaron de las licitaciones públicas para la venta de antipsicóticos, en orden decreciente según monto adjudicado. La suma total de dinero de las licitaciones en el período evaluado asciende a $\$ 4.412 .914 .162$, obteniendo los 2 primeros laboratorios: PFIZER CHILE S.A. y ASCEND LABORATORIES SPA, utilidades financieras que ascienden a $\$ 2.037 .604 .734$, representando el $46,2 \%$ del total de las compras efectuadas entre los años 2011 y 2017.

Tabla 3

Gasto público en antipsicóticos (2011 - 2017).

Laboratorios y montos de licitación (pesos chilenos)

Laboratorio Monto licitación

PFIZER CHILE S.A. 1.022 .444 .370

ASCEND LABORATORIES SPA 1.015.160.364

LABORATORIO BIOSANO S.A. 490.951 .460

LABORATORIOS ANDROMACO S.A. 402.845 .260

LABORATORIOS RECALCINE S.A. 246.311.310

LABORATORIOS SILESIA S.A.

205.874.100

BIOCROSS S.A.

197.202 .750

BPH S.A.

169.489 .920

ITF LABOMED FARMACEUTICA LTDA.

102.496 .480

ETEX FARMACEUTICA LIMITADA

90.331 .200

GLAXOSMITHKLINE CHILE FARMAC. LTDA

86.816 .640

PHARMA INVESTI DE CHILE S.A.

79.156 .350

LABORATORIO SANDERSON S.A.

76.155 .200 
NOVARTIS CHILE S.A.

72.474 .480

SEVEN PHARMA CHILE SPA

66.884 .335

LABORATORIO MAVER S.A.

50.000 .580

GEAMED S.A.

25.004 .733

ACRUX LABS S.A.

13.314 .630

Total

4.412.914.162

Nota: Elaboración propia en base a datos obtenidos de la Central Nacional de Abastecimiento (CENABAST), Ministerio de Salud, Chile (Monto neto, no incluye impuesto al valor agregadoIVA ni comisión de CENABAST).

A partir de estos resultados, se observa que en el caso de los antidepresivos, si bien hay un menor número de laboratorios que se adjudicaron compras públicas en comparación a los antipsicóticos, se observa una mayor distribución de las ganancias económicas entre las empresas participantes. Sin embargo, a pesar de ello, más de la mitad del dinero público invertido en antidepresivos se distribuye en cuatro empresas farmacéuticas. Por el contrario, en el caso de los antipsicóticos, si bien hay un mayor número de laboratorios participantes en las transacciones de compra en comparación a los antidepresivos, se presenta una mayor concentración de las ganancias en sólo dos empresas farmacéuticas durante el período analizado. Lo anterior, se explica por la compra a gran escala que realiza la CENABAST en cada proceso de licitación, y por el estrecho margen de competitividad que posee el mercado farmacéutico chileno.

Si bien estas cifras no representan la totalidad del gasto público en psicofármacos, dan cuenta que en Chile no existe una retirada del Estado y una disminución de la inversión pública en salud mental en el período 2011-2017, al menos en la compra de medicamentos psiquiátricos a través de la CENABAST. Por el contrario, esta entidad tiene un carácter relevante en la compra y distribución de psicofármacos para la 
atención ambulatoria en la red pública de salud. De esta forma, en este ámbito específico y particular, se han descrito las condiciones bajo las cuales la venta de psicofármacos por parte del sector privado es incentivada y patrocinada por el Estado, configurando un rol central para el tratamiento farmacológico en la atención de salud mental pública.

\section{Palabras finales}

Entre los años 2011 y 2017, la CENABAST intermedió la compra de $\$ 11.835 .992 .658$ en fármacos antidepresivos y antipsicóticos en Chile. Esta inversión monetaria, permite visualizar la orientación del gasto público en torno a las necesidades e intereses de la industria farmacéutica, así como la obtención de beneficios económicos de este sector empresarial con recursos estatales y la profundización del mercado en el campo de la salud mental en base a la transferencia de recursos públicos al sector privado como elemento clave de la lógica neoliberal en la gestión sanitaria.

Junto con ello, la compra de psicofármacos por parte del Estado garantiza su distribución y consumo en el sistema público de salud, legitima el acceso a estos productos farmacéuticos por parte de la ciudadanía y facilita que las personas que reciben atención de salud mental se conviertan en consumidores de estos medicamentos. En este marco, la psiquiatría de orientación biológica con sus credenciales médicas ha otorgado un manto respetable y supuestamente objetivo al abordaje farmacológico en salud mental, promoviendo exitosamente el consumo de psicofármacos en las sociedades contemporáneas (Moncrieff, 2006; Davies, 2017). Al respecto, es relevante analizar críticamente la articulación del mercado farmacéutico y la promoción de psicofármacos en un contexto neoliberal, así como el rol del Estado respecto a la alianza del gremio psiquiátrico y la industria farmacéutica, en particular, las estrategias políticas e institucionales bajo las cuales han distorsionado la evidencia 
científica, exagerando sistemáticamente los beneficios de los psicofármacos y oscureciendo sus efectos adversos (Whitaker \& Cosgrove, 2015).

Bajo estas condiciones, el aumento del consumo de medicamentos psiquiátricos representa un grave problema de salud pública. Al respecto, Peter Gøtzsche (2015) refiere que más de medio millón de personas mayores de 65 años, mueren como consecuencia del uso de fármacos psiquiátricos en el mundo occidental, cada año. Esto hace que los psicofármacos sean la tercera causa de muerte, después de las enfermedades cardíacas y el cáncer. A partir de esta constatación, Gøtzsche (2015) ha señalado que es posible reducir el consumo actual de psicofármacos en un $98 \%$ y, al mismo tiempo, aumentar los niveles de bienestar subjetivo con apoyos psicosociales y comunitarios. Asimismo, Whitaker (2015) ha descrito en qué medida el consumo de psicofármacos a largo plazo no sólo empeoran la calidad de vida y acortan la esperanza de vida de las personas, sino que también elevan las cifras de discapacidad en los países desarrollados. De acuerdo a este marco de análisis, la inversión estatal en psicofármacos representa para las arcas públicas un gasto perjudicial, marcado por la ausencia de beneficios y que acentúa un daño acumulativo, afectando directamente el bienestar de la ciudadanía.

Para afrontar este panorama, adquiere relevancia el concepto de prevención cuaternaria en salud mental, definido como las prácticas orientadas a evitar o atenuar las consecuencias de la actividad innecesaria o excesiva del sistema sanitario, en la lógica de prevenir o disminuir la incidencia de iatrogenia en la población, en este caso, el consumo ampliado y prolongado de psicofármacos (Ortiz-Lobo \& Ibáñez-Rojo, 2011). Bajo esta perspectiva, los actores sociales vinculados a los sistemas de salud mental y el conjunto de la ciudadanía, deben tomar conciencia de los graves perjuicios que pueden causar los fármacos psiquiátricos, en la perspectiva de adoptar medidas para minimizar ese daño. Al respecto, es necesario articular acciones consistentes y progresivas destinadas a disminuir radicalmente el gasto público en psicofármacos y 
reorientar esos recursos hacia servicios sociales, comunitarios y participativos, con el objetivo de contrarrestar las injusticias y desigualdades que generan malestar en el campo de la subjetividad en un contexto neoliberal.

El posicionamiento anteriormente descrito, es esencial para reflexionar respecto a un horizonte de autonomía: la posibilidad que las comunidades construyan por sí mismas una vida mejor y puedan abordar sus propias dificultades subjetivas a partir de grupos de pares, fortaleciendo los vínculos sociales, el apoyo mutuo y las luchas colectivas, sin mediar por los mecanismos del mercado farmacéutico y el consumo de psicofármacos. El camino hacia ese horizonte, implica desarrollar una mirada crítica hacia el Estado neoliberal, en particular, las prácticas y discursos bajo los cuales la institucionalidad traduce las demandas de bienestar de la ciudadanía a los parámetros del mercado y favorece el negocio de la industria farmacéutica con recursos públicos. Frente a ello, la respuesta es promover procesos de participación ciudadana y favorecer el diálogo real sobre alternativas a los psicofármacos. Más participación comunitaria y más democracia desde las bases sociales es la alternativa al neoliberalismo en salud mental.

\section{Referencias}

Alvarado, R. (2006). Evaluación de la Implementación de la Guía Clínica GES para primer episodio de esquizofrenia. Santiago de Chile: Centro de Salud Mental (CESAM).

Bentall, R. (2011). Medicalizar la mente ¿Sirven de algo los tratamientos psiquiátricos? Barcelona: Herder.

Cipriano, P. (2017). El manicomio químico. Crónicas de un psiquiatra recalcitrante. Madrid: Enclave de Libros. 
Centro Nacional de Farmacoeconomía [CENAFAR]. (2013). Medicamentos en Chile: revisión de la evidencia del mercado nacional de fármacos. Santiago: Instituto de Salud Pública.

Davies, J. (Ed.). (2017). The Sedated Society. The Causes and Harms of our Psychiatric Drug Epidemic. London: Palgrave Macmillan.

Errázuriz, P., Valdés, C., Vöhringer, P. \& Calvo, E. (2015). Financiamiento de la salud mental en Chile: una deuda pendiente. Revista médica de Chile, 143(9), 11791186

Figueroa, J. P. (2015). Farmacia municipal de Recoleta desnuda cómo la industria infla los precios de los medicamentos. Recuperado de http://ciperchile.cl/2015/11/05/farmacia-municipal-de-recoleta-desnuda-comola-industria-infla-los-precios-de-los-medicamentos/

Galleguillos, T., Risco, L., Garay, J. L., González, M. \& Vogel, M. (2003). Tendencia del uso de benzodiazepinas en una muestra de consultantes en atención primaria. Revista médica de Chile, 131(5), 535-540.

González-Guzmán, R. (2015). Ética neoliberal y nuevas ideas críticas en salud. En C. Tetelboin y A.S. Laurell (comps.). Por el derecho universal a la salud: una agenda latinoamericana de análisis y lucha (pp. 139-155). Buenos Aires: CLACSO.

Gøtzsche, P. (2015). Psicofármacos que matan y denegación organizada. Madrid: Libros del Lince.

Jirón, M., Machado, M. \& Ruiz, I. (2008). Consumo de antidepresivos en Chile entre 1992 y 2004. Revista médica de Chile, 136(9), 1147-1154.

Laval, C. y Dardot, P. (2013). La nueva razón del mundo: Ensayo sobre la sociedad neoliberal. Barcelona: Gedisa.

Ministerio de Salud (2010). Encuesta Nacional de Salud 2009-2010. Santiago: MINSAL, Gobierno de Chile. 
Miranda, G., Alvarado, S. \& Kaufman, J. S. (2012). Duración de las licencias médicas FONASA por trastornos mentales y del comportamiento. Revista médica de Chile, 140(2), 207-213.

Moncrieff, J. (2006). Psychiatric drug promotion and the politics of neo-liberalism. British Journal of Psychiatry, (188), 301-302.

Moncrieff, J. (2013). Hablando claro. Una introducción a los fármacos psiquiátricos. Barcelona: Herder.

Olivera, M. (2009). Dependencia a benzodiazepinas en un centro de atención primaria de salud: Magnitud del problema y orientaciones para el manejo integral. Revista chilena de neuro-psiquiatría, 47(2), 132-137.

Ortiz-Lobo, A. \& Ibáñez-Rojo, V. (2011). latrogenia y prevención cuaternaria en salud mental. Revista Española de Salud Pública, 85(6), 513-525

Pérez-Soto, C. (2012). Una nueva antipsiquiatría. Crítica y conocimiento de las técnicas de control psiquiátrico. Santiago: Lom.

Pérez-Soto, C. (2014). Marxismo aquí y ahora. Santiago: Editorial Triángulo.

Pérez-Soto, C. (2015). La democracia como dictadura. Athenea Digital. Revista De Pensamiento e Investigación Social, 15(4), 279-303.

Rojas, G., Fritsch, R., Galleguillos, T., Gaete, J., \& Araya, R. (2004). Consumo de psicofármacos en la población general del Gran Santiago, Chile. Revista de psiquiatría clínica, 41(2), 15-24.

Santibáñez, F. (2013). Tendencia de uso de Psicotrópicos y Estupefacientes en Chile entre el periodo 2007- 2012. (Memoria para optar al Título de Químico Farmacéutico inédita). Departamento de Ciencias y Tecnología Farmacéutica, Facultad de Ciencias Químicas y Farmacéuticas, Universidad de Chile, Santiago de Chile.

Whitaker, R. (2015). Anatomía de una epidemia. Medicamentos psiquiátricos y el asombroso aumento de las enfermedades mentales. Madrid: Capitán Swing. 
Whitaker, R. \& Cosgrove, L. (2015). Psychiatry under the influence: Institutional corruption, social injury, and prescriptions for reform. New York: Palgrave Macmillan.

Formato de citación

Cea-Madrid, J.C. (2018). Estado neoliberal y gasto público en psicofármacos en el Chile contemporáneo. Psicología, Conocimiento y Sociedad, 8(2), 50-70. doi: http://dx.doi.org/10.26864/PCS.v8.n2.4 